\title{
COMMUTING AND CENTRALIZING MAPPINGS IN PRIME RINGS
}

\author{
J. VUKMAN \\ (Communicated by Maurice Auslander) \\ Dedicated to the memory of my father
}

\begin{abstract}
Let $R$ be a ring. A mapping $F: R \rightarrow R$ is said to be commuting on $R$ if $[F(x), x]=0$ holds for all $x \in R$. The main purpose of this paper is to prove the following result, which generalizes a classical result of E. Posner: Let $R$ be a prime ring of characteristic not two. Suppose there exists a nonzero derivation $D: R \rightarrow R$, such that the mapping $x \mapsto[D(x), x]$ is commuting on $R$. In this case $R$ is commutative.
\end{abstract}

\section{Preliminaries}

Throughout this paper $R$ will represent an associative ring with center $Z(R)$. We write $[x, y]$ for $x y-y x$, and use the identities $[x y, z]=[x, z] y+x[y, z]$, $[x, y z]=[x, y] z+y[x, z]$. Recall that $R$ is prime if $a R b=(0)$ implies that either $a=0$ or $b=0$, and is semiprime if $a R a=(0)$ implies $a=0$. An additive mapping $D$ from $R$ to $R$ is called a derivation if $D(x y)=D(x) y+$ $x D(y)$ holds for all $x, y \in R$. An additive mapping $D$ from $R$ to $R$ is called a Jordan derivation if $D\left(x^{2}\right)=D(x) x+x D(x)$ is fulfilled for all $x \in R$. Obviously, every derivation is a Jordan derivation. The converse is in general not true. A well-known result first proved by I. N. Herstein [5] states that every Jordan derivation on a prime ring of characteristic not two is a derivation. A brief proof of Herstein's result can be found in [2]. A derivation $D$ is inner if there exists $a \in R$, such that $D(x)=[a, x]$ holds all for all $x \in R$. A mapping $F$ from $R$ to $R$ is said to be commuting on $R$ if $[F(x), x]=0$ for all $x \in R$, and is said to be centralizing on $R$ if $[F(x), x] \in Z(R)$ holds for all $x \in R$. There has been considerable interest in commuting, centralizing, and related mappings in prime and semiprime rings (see [1, 3, 4, 6, 7, 8, and 10] where further references can be found). Our methods are somewhat different from those employed by other authors.

Received by the editors April 18, 1989 and, in revised form, July 7, 1989.

1980 Mathematics Subject Classification (1985 Revision). Primary 16A12, 16A68, 16 A72.

Key words and phrases. Prime ring, derivation, Jordan derivation, inner derivation, commuting mapping, centralizing mapping. 


\section{The Results}

We shall need the following well-known and frequently used lemmas.

Lemma 1 [9, Lemma 1]. Let $D: R \rightarrow R$ be a derivation, where $R$ is a prime ring. Suppose that either

$$
\text { (i) } a D(x)=0, \quad x \in R
$$

or

$$
\text { (ii) } D(x) a=0, \quad x \in R
$$

holds. In both cases we have $a=0$ or $D=0$.

Lemma 2 [9, Lemma 3]. Let $D: R \rightarrow R$ be a nonzero derivation, where $R$ is a prime ring. Suppose that $D$ is commuting on $R$. In this case $R$ is commutative.

We shall start our investigations with our main result.

Theorem 1. Let $R$ be a noncommutative prime ring of characteristic not two. Suppose there exists a derivation $D: R \rightarrow R$, such that the mapping $x \mapsto$ $[D(x), x]$ is commuting on $R$. In this case $D=0$.

A classical result in the theory of centralizing mappings is a theorem of E. Posner [9, Theorem 2] which states that the existence of a nonzero centralizing derivation on a prime ring forces the ring to be commutative (obviously, Lemma 2 is a special case of this result). Neglecting the fact that in the above result we have an additional assumption concerning the characteristic of the ring, we can say, that Theorem 1 generalizes Posner's theorem.

Proof of Theorem 1. We have

$$
[[D(x), x], x]=0, \quad x \in R .
$$

Let us introduce a mapping $B(\cdot, \cdot): R \times R \rightarrow R$ by the relation

$$
B(x, y)=[D(x), y]+[D(y), x], \quad x, y \in R \text {. }
$$

It is obvious that $B(\cdot, \cdot)$ is symmetric (i.e. $B(x, y)=B(y, x)$ for all $x, y \in$ $R$ ) and additive in both arguments. Moreover, a simple calculation shows that the relation

$$
B(x y, z)=B(x, z) y+x B(y, z)+D(x)[y, z]+[x, z] D(y)
$$

is fulfilled for all $x, y, z \in R$. We introduce also a mapping $f$ from $R$ to $R$ by $f(x)=B(x, x)$. We have

$$
f(x)=2[D(x), x], \quad x \in R .
$$

Obviously, the mapping $f$ satisfies the relation

$$
f(x+y)=f(x)+f(y)+2 B(x, y), \quad x, y \in R .
$$

Throughout the proof we shall use the mapping $B(\cdot, \cdot)$ and the relations (2), (3), and (4) without specific reference. The relation (1) can now be written in the form

$$
[f(x), x]=0, \quad x \in R .
$$


The linearization of $(5)$ gives

(6) $[f(x), y]+[f(y), x]+2[B(x, y), x]+2[B(x, y), y]=0, \quad x, y \in R$.

The substitution $-x$ for $x$ in the above relation leads to

(7) $[f(x), y]-[f(y), x]+2[B(x, y), x]-2[B(x, y), y]=0, \quad x, y \in R$.

From (6) and (7) we obtain

$$
[f(x), y]+2[B(x, y), x]=0, \quad x, y \in R .
$$

Let us replace in (8) $y$ by $x y$. Then

$$
\begin{aligned}
0= & {[f(x), x y]+2[B(x y, x), x] } \\
= & {[f(x), x y]+2[f(x) y+x B(x, y)+D(x)[y, x], x] } \\
= & {[f(x), x] y+x[f(x), y]+2[f(x), x] y+2 f(x)[y, x]+2 x[B(x, y), x] } \\
& +2[D(x), x][y, x]+2 D(x)[[y, x], x]=0 .
\end{aligned}
$$

Using in the above calculation (5) and (8) we arrive at

$$
3 f(x)[y, x]+2 D(x)[[y, x], x]=0, \quad x, y \in R .
$$

Similarly, we obtain the relation

$$
3[y, x] f(x)+2[[y, x], x] D(x)=0, \quad x, y \in R
$$

putting in (8) $y x$ instead of $y$. We intend to prove that

$$
3 f(x) D(x)-D(x) f(x)=0, \quad x \in R
$$

holds. For this purpose we write $y z$ instead of $y$ in (9). We have

$$
\begin{aligned}
0= & 3 f(x)[y z, x]+2 D(x)[[y z, x], x] \\
= & 3 f(x)[y, x] z+3 f(x) y[z, x]+2 D(x)[[y, x], x] z \\
& +4 D(x)[y, x][z, x]+2 D(x) y[[z, x], x] .
\end{aligned}
$$

By (9) the above calculation reduces to

$3 f(x) y[z, x]+4 D(x)[y, x][z, x]+2 D(x) y[[z, x], x]=0, \quad x, y, z \in R$.

Putting in the above relation $y=D(x)$ we obtain $3 f(x) D(x)[z, x]+2 D(x)$ $\times f(x)[z, x]+2 D(x)^{2}[[z, x], x]=0, x, z \in R$, which yields $3 f(x) D(x)[z, x]$ $-D(x) f(x)[z, x]=0, x, z \in R$ according to (9). In other words we have proved the relation

$$
(3 f(x) D(x)-D(x) f(x))[y, x]=0, \quad x, y \in R .
$$

Now we are ready for the proof of (11). There is nothing to prove if $x \in Z(R)$, since in this case $f(x)=0$. Hence we can restrict our attention on the case $x \notin Z(R)$. In this case $y \mapsto[x, y]$ is a nonzero inner derivation, which means that from (12) and Lemma 1 it follows $3 f(x) D(x)-D(x) f(x)=0$. Thus the relation $(11)$ is proved. Similarly one proves the relation

$$
3 D(x) f(x)-f(x) D(x)=0, \quad x \in R
$$


starting from (10). From (11) and (13) one obtains easily that

$$
D(x) f(x)=f(x) D(x)=0, \quad x \in R
$$

holds. The linearization of the relation $D(x) f(x)=0$ gives $0=(D(x)+D(y))$ $\times(f(x)+f(y)+2 B(x, y))=D(x) f(x)+D(y) f(x)+D(x) f(y)+D(y) f(y)+$ $2 D(x) B(x, y)+2 D(y) B(x, y)$ which reduces to

$$
D(x) f(y)+D(y) f(x)+2 D(x) B(x, y)+2 D(y) B(x, y)=0, \quad x, y \in R \text {. }
$$

The substitution $-x$ for $x$ in (15) gives

$$
-D(x) f(y)+D(y) f(x)+2 D(x) B(x, y)-2 D(y) B(x, y)=0, \quad x, y \in R .
$$

Combining (15) with (16) we arrive at

$$
D(y) f(x)+2 D(x) B(x, y)=0, \quad x, y \in R .
$$

Put in (17) $y x$ for $y$. Then $0=D(y x) f(x)+2 D(x) B(y x, x)=D(y) x f(x)+$ $y D(x) f(x)+2 D(x) B(y, x) x+2 D(x) y f(x)+2 D(x)[y, x] D(x)$ which leads to $D(y) x f(x)+2 D(x) B(x, y) x+2 D(x) y f(x)+2 D(x)[y, x] D(x)=0, \quad x, y \in R$ according to (14). The relation (17) makes us possible to write $-D(y) f(x)$ instead of $2 D(x) B(x, y)$ in the above relation. Thus we have

$$
D(y)[x, f(x)]+2 D(x) y f(x)+2 D(x)[y, x] D(x)=0,
$$

which yields

$$
D(x) y f(x)+D(x)[y, x] D(x)=0, \quad x, y \in R
$$

according to (5). Let us write in (18) $x y$ for $y$. Then $0=D(x) x y f(x)+$ $D(x)[x y, x] D(x)=D(x) x y f(x)+D(x) x[y, x] D(x)$. Thus we have

$$
D(x) x y f(x)+D(x) x[y, x] D(x)=0, \quad x, y \in R .
$$

Left multiplication of the relation (18) by $x$ gives

$$
x D(x) y f(x)+x D(x)[y, x] D(x)=0, \quad x, y \in R .
$$

Combining (19) with (20) we arrive at

$$
f(x) y f(x)+f(x)[y, x] D(x)=0, \quad x, y \in R .
$$

Our next step is to prove the relation

$$
3 f(x) y f(x)+4 f(x)[y, x] D(x)=0, \quad x, y \in R .
$$

For this purpose we write in (10) $y z$ instead of $y$. We have $0=3[y z, x] f(x)+$ $2[[y z, x], x] D(x)=3[y, x] z f(x)+3 y[z, x] f(x)+2[[y, x], x] z D(x)+4[y, x]$ $\times[z, x] D(x)+2 y[[z, x], x] D(x)$ which leads to

$$
3[y, x] z f(x)+2[[y, x], x] z D(x)+4[y, x][z, x] D(x)=0, \quad x, y, z \in R
$$


according to (10). Putting in (23) $y=2 D(x)$ and making use of (5) we arrive at $3 f(x) z f(x)+4 f(x)[z, x] D(x)=0, x, z \in R$ which completes the proof of (22). From (21) and (22) one obtains immediately

$$
f(x) y f(x)=0, \quad x, y \in R
$$

which implies $f(x)=0, x \in R$ by primeness of $R$. Thus we have proved that $[D(x), x]=0$ holds for all $x \in R$, which yields $D=0$ by Lemma 2 . The proof of the theorem is complete.

We are ready for our next result.

Theorem 2. Let $R$ be a noncommutative prime ring of characteristic different from two and three. Suppose there exists a derivation $D: R \rightarrow R$, such that the mapping $x \mapsto[D(x), x]$ is centralizing on $R$. In this case $D=0$.

Proof. Throughout the proof we shall use the same notation as in the proof of Theorem 1. The assumption of the theorem can be written as follows

$$
[f(x), x] \in Z(R), \quad x \in R .
$$

Using similar approach as in the proof of (8) we obtain from (24) that the relation

$$
[f(x), y]+2[B(x, y), x] \in Z(R), \quad x, y \in R
$$

is fulfilled. Putting in (25) $x^{2}$ for $y$ we obtain $\left[f(x), x^{2}\right]+2[f(x) x+x f(x), x]$ $\in Z(R), x \in R$, which yields $[f(x), x] x+x[f(x), x]+2[f(x), x] x+$ $2 x[f(x), x] \in Z(R), x \in R$. Hence

$$
6[f(x), x] x \in Z(R), \quad x \in R .
$$

From (24) and (26) we conclude that $6[f(x), x][x, y]=0$ holds for all $x, y \in$ $R$, which leads to

$$
[f(x), x][x, y]=0, \quad x, y \in R
$$

since we have assumed that $R$ is of characteristic different from two and three. We intend to prove that

$$
[f(x), x]=0, \quad x \in R
$$

is true. Obviously, we can restrict our attention on the case when $x \notin Z(R)$. For any fixed $x \notin Z(R)$, a mapping $y \mapsto[x, y]$ is a nonzero inner derivation, which means that (27) and Lemma 1 imply $[f(x), x]=0$. Since all the requirements of Theorem 1 are fulfilled, we conclude that $D=0$. The proof of the theorem is complete.

It would be interesting to know whether Theorem 2 can be proved without the assumption that $R$ is of characteristic different from three. Theorem 1 will be used in the proof of our last result. 
Theorem 3. Let $R$ be a noncommutative prime ring of characteristic different from two and three. Suppose $R$ contains the identity element 1 . Let $D: R \rightarrow R$ be an additive mapping, such that $D\left(x^{3}\right)=3 x D(x) x$ holds for all $x \in R$. In this case $D=0$.

Proof. From

$$
D\left(x^{3}\right)=3 x D(x) x, \quad x \in R
$$

it follows immediately

$$
D(1)=0 \text {. }
$$

Putting in (29) $x+1$ instead of $x$, and making use of (29) and (30), one obtains easily that $3 D\left(x^{2}\right)=3 D(x) x+3 x D(x), x \in R$ holds. Since we have assumed that $R$ is of characteristic different from three, we have $D\left(x^{2}\right)=$ $D(x) x+x D(x), x \in R$. In other words, $D$ is a Jordan derivation. We know that any Jordan derivation on a prime ring of characteristic not two is a derivation. One can replace in (29) $D\left(x^{3}\right)$ by $D(x) x^{2}+x D(x) x+x^{2} D(x)$, which reduces (29) to $D(x) x^{2}+x^{2} D(x)-2 x D(x) x=0, x \in R$. This relation can be written in the form

$$
[[D(x), x], x]=0, \quad x \in R .
$$

Therefore all the assumptions of Theorem 1 are fulfilled, which means that $D=0$. The proof of the theorem is complete.

\section{ACKNOWLEDGMENT}

I wish to express my thanks to the referee for helpful suggestions.

\section{REFERENCES}

1. H. E. Bell and W. S. Martindale, Centralizing mappings of semiprime rings, Canad. Math. Bull. 30 (1) (1987), 92-101.

2. M. Brešar and J. Vukman, Jordan derivations on prime rings. Bull. Austral. Math. Soc. 37 (1988), 321-322.

3. __ On left derivations and related mappings Proc. Amer. Math. Soc. (to appear).

4. $\ldots$, On some additive mappings in rings with involution, Aequationes Math. 38 (1989), $178-185$.

5. I. N. Herstein, Jordan derivations in prime rings, Proc. Amer. Math. Soc. 8 (1957), 11041110.

6. J. H. Mayne, Centralizing automorphisms of prime rings, Canad. Math. Bull. 19 (1) (1976), 113-115.

7. __ Ideals and centralizing mappings in prime rings, Proc. Amer. Math. Soc. 86 (1982), 211-212; Erratum 89 (1983), 198.

8. ___ Centralizing mappings of prime rings, Canad. Math. Bull. 27 (1) (1984), 122-126.

9. E. Posner, Derivations in prime rings, Proc. Amer. Math. Soc. 8 (1957), 1093-1100.

10. J. Vukman, Symmetric bi-derivations on prime and semi-prime rings, Aequationes Math. 38 (1989) 245-254.

University of Maribor, VEKŠ, Razlagova 14, 62000 Maribor, Yugoslavia 